\title{
High-mass star formation in the IRAS 17233-3606 region: a new nearby and bright hot core in the southern sky ${ }^{\star}$
}

\author{
S. Leurini ${ }^{1,2}$, C. Hieret $^{2}$, S. Thorwirth ${ }^{2}$, F. Wyrowski ${ }^{2}$, P. Schilke ${ }^{2}$, K. M. Menten ${ }^{2}$, R. Güsten ${ }^{2}$, and L. Zapata ${ }^{2}$ \\ 1 ESO, Karl-Scharzschild-Strasse 2, 85748 Garching-bei-München, Germany \\ e-mail: sleurini@eso.org \\ 2 Max Planck Institut für Radioastronomie, Auf dem Hügel 69, 53121 Bonn, Germany \\ e-mail: name@mpifr-bonn.mpg.de
}

Received 28 January 2008 / Accepted 27 April 2008

ABSTRACT

\begin{abstract}
Aims. We present molecular line observations of the massive star-forming region IRAS 17233-3606 aimed at studying the molecular core associated with the source.

Methods. The observations were made using the Atacama Pathfinder Experiment telescope in the $\mathrm{CO}(3-2)$ and $\mathrm{HCO}^{+}(4-3)$ transitions and in the $\mathrm{CH}_{3} \mathrm{OH}\left(6_{K}-5_{K}\right),\left(7_{K}-6_{K}\right)$, and $\mathrm{CH}_{3} \mathrm{CN}\left(16_{K}-15_{K}\right)$ bands. For the $\mathrm{CO}(3-2)$ and $\mathrm{HCO}^{+}(4-3)$ transitions, we obtained maps with a size of $70^{\prime \prime} \times 70^{\prime \prime}$. The typical angular resolution of the data is $\sim 18^{\prime \prime}$.

Results. Our observations reveal an exceptionally rich molecular spectrum, one signpost of hot core activity. Comparisons with two other prominent southern hot cores were made through observations in the same frequency setups. We also detected a bipolar outflow in $\mathrm{CO}(3-2)$ and $\mathrm{HCO}^{+}(4-3)$ lines. Modelling reveals a hot core of size $\sim 3^{\prime \prime}$ and a temperature of $150 \mathrm{~K}$ in the IRAS $17233-3606$ region. The parameters of the molecular outflow are derived through the analysis of the $\mathrm{CO}(3-2)$ emission, and are typical of outflows driven by high-mass young stellar objects.
\end{abstract}

Key words. ISM: jets and outflows - ISM: molecules - stars: individual: IRAS 17233-3606 - stars: formation

\section{Introduction}

The last decade has seen significant progress in the understanding of how high-mass stars form. Large samples of massive young stellar objects (YSOs) were studied with single-dish telescopes to investigate their physical properties through the analysis of their (sub)mm continuum and molecular emission (e.g., Molinari et al. 1996, 1998, 2000; Walsh et al. 1997, 1998, 1999; Hatchell et al. 2000; Zhang et al. 2001, 2005; Sridharan et al. 2002; Beuther et al. 2002b,c; Faúndez et al. 2004; Williams et al. 2004, 2005). However, an intrinsic feature of high-mass stars is that they form in clusters, and that most of them are at large (several kpc) distances. Therefore, single-dish studies lack the necessary spatial resolution to resolve single protostars. However, they are essential for performing statistical studies on large samples of objects, and to identify the best candidates for follow up analysis at higher resolution. Since the Atacama Large Millimeter Array will regularly operate at $0.1^{\prime \prime}$ scales in the near future, it is now important to select massive young stellar object candidates that are observable from the southern hemisphere and relatively close-by in order to reach linear resolution that is sufficient for detailed studies of the chemical and physical processes in the environment of the newly formed high-mass stars and the latter's interaction with their environment.

The region harbouring the prominent far-infrared source IRAS 17233-3606 (hereafter IRAS 17233) first came to attention through its very intense $\mathrm{H}_{2} \mathrm{O}, \mathrm{OH}$, and $\mathrm{CH}_{3} \mathrm{OH}$ masers. The combination of the latter is an unambiguous signpost of recent

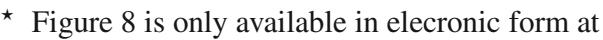
http://www . aanda.org
}

high-mass star formation (e.g., Forster \& Caswell 1989; Walsh et al. 1998). IRAS 17233, also known as G351.78-0.54, contains two cm continuum sources with a separation of $\sim 12^{\prime \prime}$, one being a compact HII region, the other a weak double UCHII source (Hughes \& MacLeod 1993) close to the location of the maser spots. Recently, higher angular resolution multi-radio wavelengths observations by Zapata et al. (2008) found a cluster of four compact radio objects of sizes equal or less than $0.3^{\prime \prime}$ and varying (mostly thermal) spectral indices in the region of the double radio source. One of them is at the centre of a bipolar outflow traced by $\mathrm{OH}$ masers (Fish et al. 2005). Moreover, nonGaussian profiles were detected in $\mathrm{CO}(2-1)$ by Osterloh et al. (1997) which are probably associated with a molecular outflow. From these data however, the powering source of the molecular outflow remains unidentified.

Previous studies seem to agree that IRAS 17233 is located at the near kinematic distance (between $700 \mathrm{pc}$ and $2.2 \mathrm{kpc}$, Miettinen et al. 2006; Forster \& Caswell 1989) rather than at the far distance ( $\sim 16 \mathrm{kpc}$, e.g. MacLeod et al. 1998). This is suggested by the high measured intensities of practically all emissions, which would indicate exceedingly high luminosities if the source were at the far distance and, persuasively, by the fact that it is at an angular distance of more than 0.5 degrees below the Galactic plane. In the following discussion, we adopt a distance of $1 \mathrm{kpc}$ for the source. Continuum observations of dust emission at $1.2 \mathrm{~mm}$ (Faúndez et al. 2004) imply a mass of $200 M_{\odot}$, and a bolometric luminosity of $2.7 \times 10^{4} L_{\odot}$ for the region follows from its spectral energy distribution. So far, little data are available on thermally excited molecular line emission from IRAS 17233, which would allow investigation of the physical and chemical status of the region and lay the groundwork for future detailed studies with ALMA. 
In this paper, we report observations of IRAS 17233 with the Atacama Pathfinder Experiment telescope ${ }^{1}$ in several molecular tracers, with typical angular resolutions of $18^{\prime \prime}$. These data reveal a bipolar outflow originating from a position very close to the methanol maser spots detected by Walsh et al. (1998), and a rich molecular spectrum typical of hot cores near massive young stellar objects.

\section{Observations}

The observations were made during 2006 April and August with the Atacama Pathfinder Experiment 12 m telescope (APEX) located on Llano de Chajnantor in the Atacama desert of Chile. These observations are part of a larger programme aimed at studying massive young stellar objects (YSOs) in the southern hemisphere (Hieret et al. 2007).

IRAS 17233 was observed with the APEX-2A facility receiver (Risacher et al. 2006) tuned at $356.7 \mathrm{GHz}$ in the upper side band, in order to simultaneously detect the $\mathrm{HCO}^{+}$(4-3) transition (and, from mapping, retrieve the peak position) as well as the $\mathrm{CO}(3-2)$ line from the lower side band (to detect any outflow activity). A map of $70^{\prime \prime} \times 70^{\prime \prime}$ with a spacing of $10^{\prime \prime}$ was taken centred on the $1.2 \mathrm{~mm}$ continuum peak position from Faúndez et al. $(2004)\left(\alpha_{2000}=17^{\mathrm{h}} 26^{\mathrm{m}} 43.0^{\mathrm{s}}, \delta_{2000}=-36^{\circ} 09^{\prime} 15.5^{\prime \prime}\right)$. These observations were followed up by ON-OFF integrations on the peak of the $\mathrm{HCO}^{+}(4-3)$ emission $\left(\alpha_{2000}=\right.$ $\left.17^{\mathrm{h}} 26^{\mathrm{m}} 42.2^{\mathrm{s}}, \delta_{2000}=-36^{\circ} 09^{\prime} 21.5^{\prime \prime}\right)$ in $\mathrm{CH}_{3} \mathrm{OH}\left(6_{K}-5_{K}, v_{t}=\right.$ $0,1)$ at $289.8 \mathrm{GHz}$ in $\mathrm{LSB}, \mathrm{CH}_{3} \mathrm{CN}\left(16_{K}-15_{K}\right)$ at $294.45 \mathrm{GHz}$ in USB, and $\mathrm{CH}_{3} \mathrm{OH}\left(7_{K}-6_{K}, v_{t}=0,1\right)$ at $337.8 \mathrm{GHz}$ in LSB. For comparison, we also observed the massive star-forming regions G327.3-0.6 $\left(\alpha_{2000}=15^{\mathrm{h}} 53^{\mathrm{m}} 08.6^{\mathrm{s}}, \delta_{2000}=-54^{\circ} 37^{\prime} 07.3^{\prime \prime}\right)$ and NGC 6334(I) $\left(\alpha_{2000}=17^{\mathrm{h}} 20^{\mathrm{m}} 53.4^{\mathrm{s}}, \delta_{2000}=-35^{\circ} 47^{\prime} 01.5^{\prime \prime}\right)$ at 289.8 and $294.45 \mathrm{GHz}$. The OFF positions were, in all cases, at $\left(600^{\prime \prime}, 0^{\prime \prime}\right)$ from the reference positions. The APEX facility FFT spectrometers (Klein et al. 2006) were used in series with $100 \mathrm{MHz}$ of overlap, thereby providing $1.8 \mathrm{GHz}$ of bandwidth. Due to the high dynamic range of the FFTS ( $48 \mathrm{~dB}$ of the 8 bit analog-to-digital converter) the IF processor had to limit the band edges with steep band pass filters to avoid aliasing by out-of-band signals. However, because of the limited steepness of any analog filter, residual aliasing is unavoidable, thereby limiting the effective bandwidth of the spectrometer. The region of the spectrum which can be affected by this problem is limited to the outer $\sim 50 \mathrm{MHz}$ of bands.

In August 2006, continuum cross-scans were performed with the APEX-2A and the FLASH receivers (Heyminck et al. 2006) at $344.7,461$ and $806.7 \mathrm{GHz}$ respectively, towards the $1.2 \mathrm{~mm}$ continuum peak position from Faúndez et al. (2004).

The pointing of the telescope was checked on Jupiter, NGC6334(I) and SGRB2-N and found to be accurate to $2^{\prime \prime}$ (rms).

The observed transitions and basic observational parameters are summarised in Tables 1 and 2. We used a main-beam efficiency of $0.73,0.60$ and 0.43 for APEX-2a, FLASH-460 and FLASH-810 respectively, to convert antenna temperatures into main-beam temperatures. A detailed description of APEX and its performance is given by Güsten et al. (2006).

\footnotetext{
1 This publication is based on data acquired with the Atacama Pathfinder Experiment (APEX). APEX is a collaboration between the Max-Planck-Institut für Radioastronomie, the European Southern Observatory, and the Onsala Space Observatory.
}

Table 1. Summary of the parameters of the APEX line observations.

\begin{tabular}{lcccc}
\hline \hline \multicolumn{1}{c}{ Line } & $\begin{array}{c}\text { Tuning Frequency } \\
(\mathrm{MHz})\end{array}$ & $\begin{array}{c}\text { Beam } \\
\left({ }^{\prime \prime}\right)\end{array}$ & $\begin{array}{c}\Delta v \\
\left(\mathrm{~km} \mathrm{~s}^{-1}\right)\end{array}$ & $\begin{array}{c}T_{\text {sys }} \\
(\mathrm{K})\end{array}$ \\
\hline $\mathrm{HCO}^{+a}(4-3)$ & 356734.3 & 18 & 0.05 & 270 \\
$\mathrm{CH}_{3} \mathrm{OH}\left(6_{K}-5_{K}\right)$ & 289125.0 & 22 & 0.06 & 293 \\
$\mathrm{CH}_{3} \mathrm{CN}\left(16_{K}-15_{K}\right)$ & 294450.0 & 21 & 0.06 & 330 \\
$\mathrm{CH}_{3} \mathrm{OH}\left(7_{K}-6_{K}\right)$ & 337800.0 & 19 & 0.05 & 160 \\
\hline a with $\mathrm{CO}(3-2)$ in the lower side band. & & &
\end{tabular}

Table 2. Summary of the parameters of the APEX continuum observations.

\begin{tabular}{ccr}
\hline \hline $\begin{array}{c}\text { Tuning Frequency } \\
(\mathrm{MHz})\end{array}$ & $\begin{array}{c}\text { Beam } \\
\left({ }^{\prime \prime}\right)\end{array}$ & \multicolumn{1}{c}{$\begin{array}{c}T_{\text {sys }} \\
(\mathrm{K})\end{array}$} \\
\hline 344770.0 & 18 & 150 \\
461040.8 & 14 & 590 \\
806651.8 & 8 & 2171 \\
\hline
\end{tabular}

\section{Observational results}

\section{1. $\mathrm{HCO}^{+}$and $\mathrm{CO}$ maps}

Figure 1a shows the spectrum of the $\mathrm{CO}(3-2)$ transition toward the peak position of the $\mathrm{HCO}^{+}$(4-3) emission. The line, while at the edge of the observed band, is only marginally affected by the aliasing out-of-band problem discussed in Sect. 2, since the $50 \mathrm{MHz}$ at the edge of the FFTS unit starts at a velocity of $-51 \mathrm{~km} \mathrm{~s}^{-1}$ where the emission in the blue-shifted wing is negligible. The line has non-Gaussian blue- and redshifted wings with respect to the systemic ambient cloud velocity $\left(-3.4 \mathrm{~km} \mathrm{~s}^{-1}\right)$, which extend to a maximum blue- and redshifted velocity of $\sim \pm 50 \mathrm{~km} \mathrm{~s}^{-1}$ respectively. In addition, two narrower lines are detected at $\sim 40$ and $58 \mathrm{~km} \mathrm{~s}^{-1}$, which could be high velocity bullets associated with the outflow, but could also be due to unidentified molecular species and be associated with the central source. The rest frequencies of these features are 345.75 and $345.72 \mathrm{GHz}$ respectively, if they come from the lower side band of the receiver, 356.25 and $356.28 \mathrm{GHz}$ if they are from the upper side band.

Broad non-Gaussian wings are also seen in the $\mathrm{HCO}^{+}$transition $\left(v_{\text {blue }}^{\max }=-38, v_{\text {red }}^{\max }=26 \mathrm{~km} \mathrm{~s}^{-1}\right)$. However, the profiles of the two transitions differ significantly from each other, with the CO line showing deep absorption features probably due to selfabsorption as well as to absorption from other unrelated foreground clouds. Figures $2 \mathrm{a}$ and $2 \mathrm{~b}$ show a map of the integrated intensity of the two transitions in the line wings.

\subsection{Molecular observations}

To investigate the physics of the molecular core in IRAS 17233 , we performed long integration observations toward the peak position of the $\mathrm{HCO}^{+}$emission in several $\mathrm{CH}_{3} \mathrm{OH}$ transitions and in the $\mathrm{CH}_{3} \mathrm{CN}\left(16_{K}-15_{K}\right)$ band, since both molecules can be used as probes of the physics of the gas (Olmi et al. 1993; Leurini et al. 2004). The flexibility of the FFTS at APEX allows the simultaneous observation of $1.8 \mathrm{GHz}$ bandwidth, with a velocity resolution down to $\sim 0.05 \mathrm{~km} \mathrm{~s}^{-1}$ at $\sim 1 \mathrm{~mm}$. For the analysis of the data, we smoothed all spectra to a resolution of $1 \mathrm{~km} \mathrm{~s}^{-1}$. In this way, we observed transitions from the first torsionally excited levels of methanol together with transitions from its ground state; lines from $\mathrm{CH}_{3}^{13} \mathrm{CN}$ and ${ }^{13} \mathrm{CH}_{3} \mathrm{OH}$ also fall in the observed bands. We could detect the $\mathrm{CH}_{3} \mathrm{CN}\left(16_{K}-15_{K}\right)$ band up to $K=7$. The identification of higher $K$ transitions in the observed 


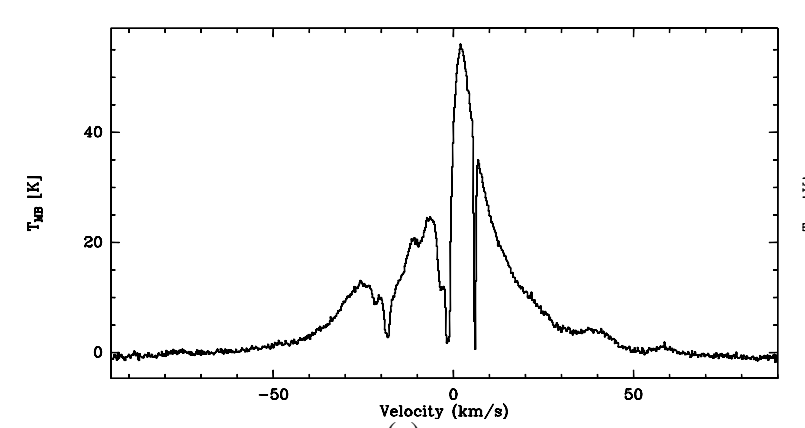

(a)

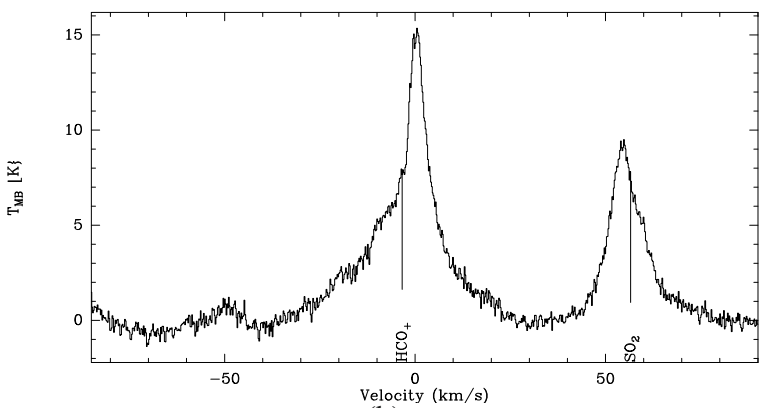

(b)

Fig. 1. $\mathrm{CO}(3-2)$ a) and $\mathrm{HCO}^{+}$(4-3) b) spectra towards the peak position of the $\mathrm{HCO}^{+}$(4-3) line (marked by a triangle in Fig. 2).

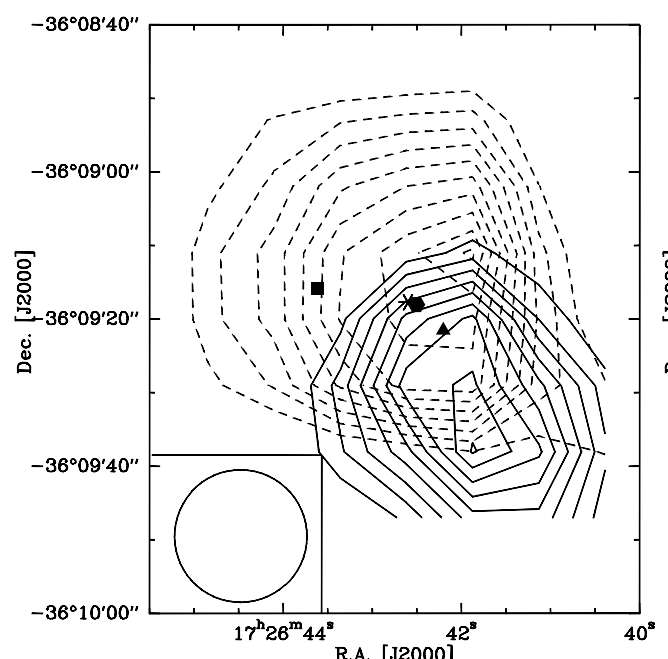

(a)

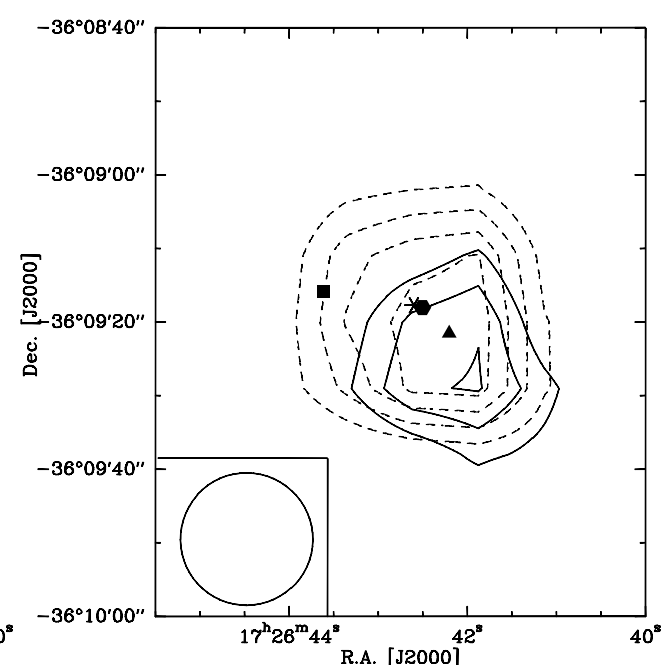

(b)

Fig. 2. a) Map of the integrated intensity of the $\mathrm{CO}(3-2)$ transition (blue-shifted emission $v=[-50,-10] \mathrm{km} \mathrm{s}^{-1}$; red-shifted emission $v=$ $[9,50] \mathrm{km} \mathrm{s}^{-1}$; levels from $70 \mathrm{~K} \mathrm{~km} \mathrm{~s}^{-1}$ in steps of 35, equivalent to $\left.\sim 5 \sigma\right)$. b) Map of the integrated intensity of the $\mathrm{HCO}^{+}(4-3)$ transition (blueshifted emission $v=[-35,-8] \mathrm{km} \mathrm{s}^{-1}$; red-shifted emission $v=[5,24] \mathrm{km} \mathrm{s}^{-1}$; levels from $20 \mathrm{~K} \mathrm{~km} \mathrm{~s}^{-1}$ in steps of 10 , equivalent to $\left.\sim 5 \sigma\right)$. In both maps dashed contours are used for the blue-shifted emission, solid contours for the red-shifted emission. The triangle marks the peak position of the $\mathrm{HCO}^{+}$emission, the square the compact HII region, the hexagon the centroid of the multiple radio continuum source, and the asterisk the position of the $\mathrm{CH}_{3} \mathrm{OH}$ maser spot (Walsh et al. 1998; Zapata et al. 2008). The beam of the APEX telescope at the observing frequency is shown in the left bottom corner.

band is rendered more difficult by their overlap with other lines. Having access to high excitation lines, like the $v_{t}=1 \mathrm{CH}_{3} \mathrm{OH}$ transitions, and to low opacity lines (e.g., the $\mathrm{CH}_{3}^{13} \mathrm{CN}$ and the $\mathrm{CH}_{3}^{13} \mathrm{OH}$ bands) guarantees a better determination of the physical parameters of the emitting gas (see Sect. 4.3).

The molecular spectrum of IRAS 17233 shows strong emission from several $\mathrm{CH}_{3} \mathrm{OH}$ and $\mathrm{CH}_{3} \mathrm{CN}$ lines, typical of hot cores; moreover, several other transitions from complex molecules are detected. For comparison, we observed the southern hot cores in NGC 6334(I) and G327.3-0.6 (e.g., Schilke et al. 2006) in the same setup of IRAS 17233 in the $\mathrm{CH}_{3} \mathrm{OH}\left(6_{K}-5_{K}\right)$ and $\mathrm{CH}_{3} \mathrm{CN}$ $\left(16_{K}-15_{K}\right)$ bands. The spectra are in general comparable, although the linewidths of IRAS $17233\left(\Delta v \sim 6-9 \mathrm{~km} \mathrm{~s}^{-1}\right)$ are larger than in the other two hot cores $\left(\Delta v \sim 3-5 \mathrm{~km} \mathrm{~s}^{-1}\right)$. Several species in IRAS $17233\left(\mathrm{CS}, \mathrm{H}_{2} \mathrm{CO}, \mathrm{SO}, \mathrm{SO}_{2}, \mathrm{HCO}^{+}\right.$, $\mathrm{CO})$ show non-Gaussian wings probably associated with the outflow. Figure 3 presents one of the observed spectral window towards the three sources.

\section{Physical parameters}

The submm continuum flux of IRAS 17233 is strong enough to do continuum cross-scans on the source. These observations found the peak emission at $\left(-6^{\prime \prime},-2^{\prime \prime}\right)$ from the position used as reference, and not coincident with the peak of the $\mathrm{HCO}^{+}$(4-3) emission (see Fig. 2) which we used for the spectroscopic observations of the hot core. Fit results from averaged cross-scans at the different frequencies are given in Table 3.

The peak position of the $\mathrm{HCO}^{+}$emission was derived by integrating the line intensity over a range of velocities close to the systemic velocity, and contamination from the molecular outflow could affect this estimate. The peak of the submmcontinuum emission is very close $\left(-3{ }^{\prime \prime} 7,0,0^{\prime} 2\right)$ to the position of the methanol maser spots detected by Walsh et al. (1998), and a weak radio continuum source (named $2 b$ by Zapata et al. 2008). With the expected pointing uncertainties the three positions are practically indistinguishable. In the following discussion, we will use the position of the the class II methanol maser spots $\left(\alpha_{2000}=17^{\mathrm{h}} 26^{\mathrm{m}} 42.6^{\mathrm{s}}, \delta_{2000}=-36^{\circ} 09^{\prime} 17.7^{\prime \prime}\right)$ as reference position for the hot core in IRAS 17233, since class II methanol masers are found only in the vicinity of massive protostars. We also assume that the hot core is the powering source of the molecular outflow and that it is associated with the UCHII region detected at cm wavelengths (Hughes \& MacLeod 1993). Furthermore, in the following discussion we will assume that the 


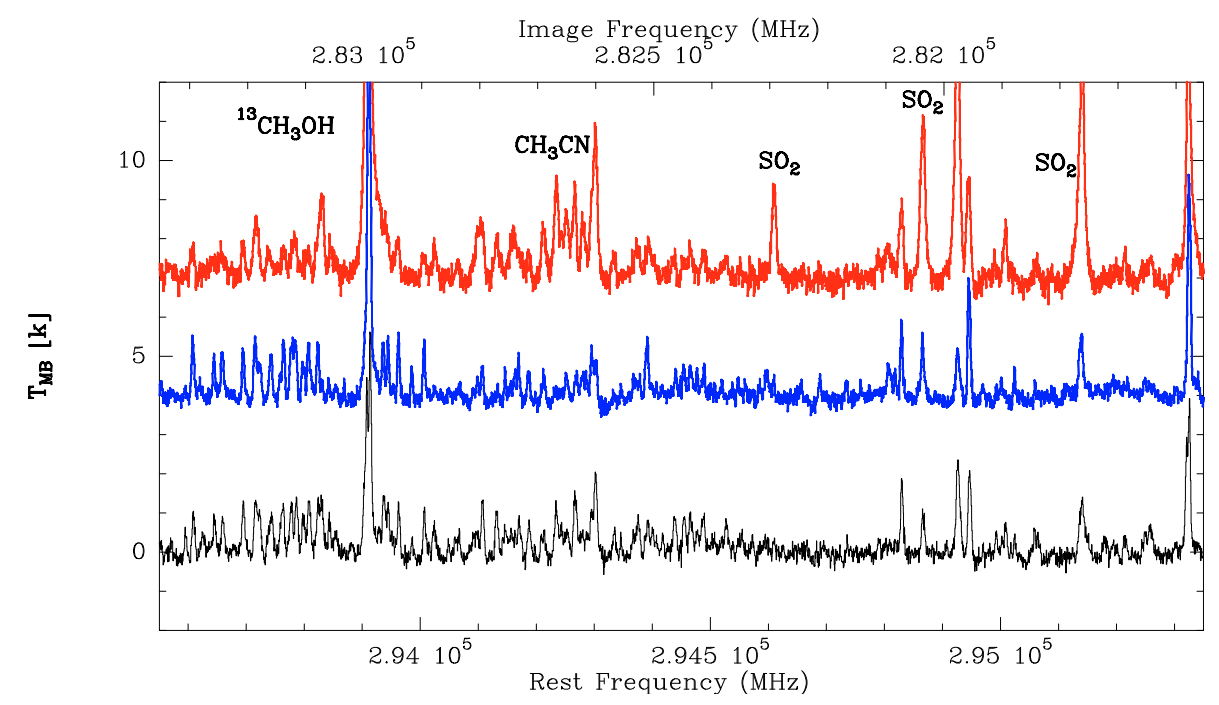

Fig. 3. Spectrum at 294.5 GHz of G327.3-0.6 (bottom), NGC 6334(I) (centre) and IRAS 17233 (top). The features labelled in the spectra are the ones discussed in Sect. 4.4. The strong feature at the right end of the bandwidth is a mirror from the lower side band of the $\mathrm{H}_{2} \mathrm{CO}\left(4_{1,4}-3_{1,3}\right)$ transition at $281.5 \mathrm{GHz}$.

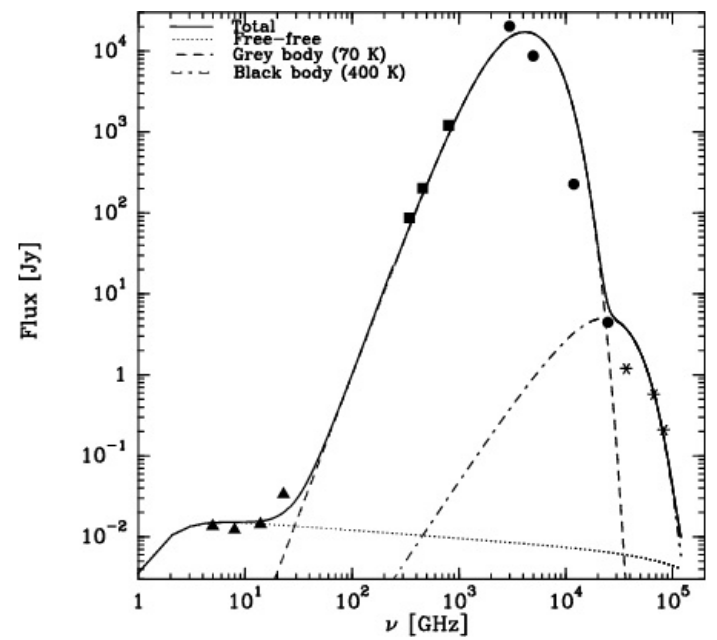

Fig. 4. Spectral energy distribution of the hot core in IRAS 17233. Observational fluxes from the VLA, APEX, IRAS and IRAC are marked as triangles, squares, circles and asterisks respectively. For illustration, the flux density curve of an ultra-compact HII region, of a $70 \mathrm{~K}$ grey body, and of a $400 \mathrm{~K}$ black body are also given. The solid black line represents the combined spectrum of the three components.

total luminosity of the region is driven by the same source (harbouring the hot core and the UCH II). The spectral type of the $\mathrm{UCH}$ II derived from its radio continuum emission is B1 (Zapata et al. 2008), while that of the HII region B0.5. However, the $850 \mu \mathrm{m}$ dust continuum map, taken during the ASTROGAL survey of the Galactic Plane with the APEX telescope (Schuller, priv. comm.), is peaked on the hot core and not on the HII region, suggesting that the IR luminosity of the source is indeed dominated by the UCHII region.

Observations at shorter wavelengths were performed by several authors: Fix et al. (1982) and Walsh et al. (1999) detected emission in the direction of the UCHII region in the near infrared $K$ and $L$ bands $(2.2 \mu \mathrm{m}$ and $3.3 \mu \mathrm{m}$ respectively), while the HII region is detected in the IR $K$ and $L$ bands and at $10 \mu \mathrm{m}$ (Fix et al. 1982; Walsh et al. 1999; De Buizer et al. 2000). The area is also covered by the GLIMPSE (Benjamin et al. 2003) survey from the Spitzer Space Telescope, whose point source
Table 3. Summary of the APEX continuum observations.

\begin{tabular}{crccr}
\hline $\begin{array}{c}\text { Frequency } \\
(\mathrm{GHz})\end{array}$ & $\begin{array}{c}T_{\mathrm{MB}} \\
(\mathrm{K})\end{array}$ & $\begin{array}{c}\text { Obs. size } \\
\left({ }^{\prime \prime}\right)\end{array}$ & $\begin{array}{c}\text { Dec. size } \\
\left({ }^{\prime \prime}\right)\end{array}$ & $\begin{array}{c}\text { Flux density } \\
(\mathrm{Jy})\end{array}$ \\
\hline 347.7 & 1.8 & 22.1 & 12.7 & 88 \\
461.0 & 3.6 & 17.9 & 12.0 & 204 \\
806.7 & 10.7 & 14.6 & 12.4 & 1214 \\
\hline
\end{tabular}

catalogue has an astrometric accuracy of $0.3^{\prime \prime}$. No emission is detected in the $3.6,4.5$ or $8.0 \mu \mathrm{m}$ bands towards the hot core, but all bands are dominated by strong emission from a source to the north-east of the hot core, not associated with any cm continuum emission. Emission in the 3.6 and $4.5 \mu \mathrm{m}$ bands is also found close to the hot core at $\left(-4.8^{\prime \prime}, 0.2^{\prime \prime}\right)$. The MIPSGAL survey also covers this area (Carey et al. 2005), but both bands ( 24 and $70 \mu \mathrm{m}$ ) are saturated. However, by comparing the emission at $24 \mu \mathrm{m}$ (and 70) with that at $8 \mu \mathrm{m}$, the peak of the intensity in the MIPS bands seems to be shifted towards the hot core position as at $\mathrm{mm}$ wavelengths.

\subsection{Continuum}

The mass of the core can be derived from the measured flux at submm wavelengths, since in this part of the spectrum the continuum emission is dominated by thermal dust emission. However, the measurements at 461 and $806.7 \mathrm{GHz}$ should be regarded as upper limits to the continuum emission since they are likely to be contaminated by emission from the $\mathrm{CO}(4-3)$ and (7-6) lines respectively. Assuming a distance of $1 \mathrm{kpc}$ for the source, and a temperature of $70 \mathrm{~K}$, the flux at $347.7 \mathrm{GHz}$ converts into a mass for the core of $107 M_{\odot}$ for a dust emissivity of $1.8 \mathrm{~cm}^{2} \mathrm{~g}^{-1}$ (Ossenkopf \& Henning 1994), or $130 M_{\odot}$ for a standard Hildebrand (1983) opacity with $\beta=1.5$. The value obtained by Faúndez et al. (2004) was $210 M_{\odot}$, for $T_{\mathrm{d}}=45 \mathrm{~K}$ and $d=800$ pc.

In Fig. 4, we show the spectral energy distribution of IRAS 17233 from $5 \mathrm{GHz}$ to the near IR. Given our resolution, several of the IRAC sources in the region could coincide with the hot core giving rise to the submm emission or none at all. Since we assume that the hot core coincides with the $\mathrm{CH}_{3} \mathrm{OH}$ maser, 


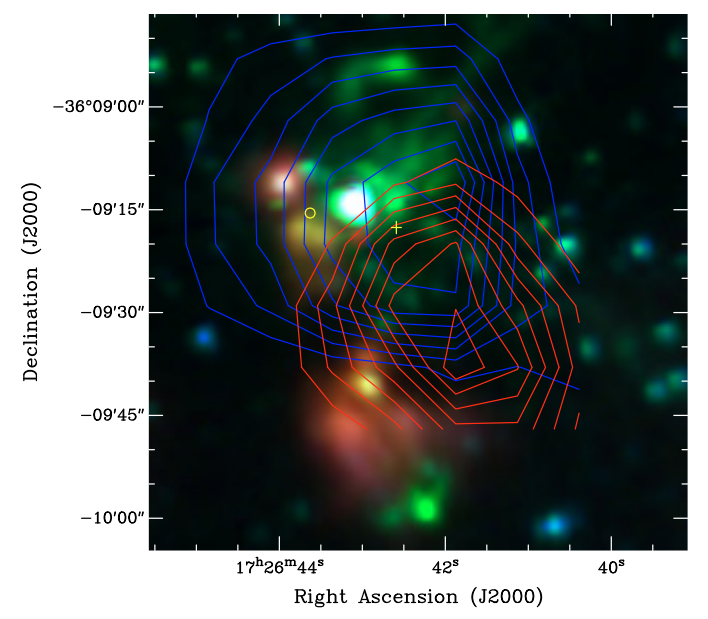

Fig. 5. Spitzer image of the IRAS 17233-3606 region; the colour scheme is the following: $3.6 \mu \mathrm{m}$ (blue), $4.5 \mu \mathrm{m}$ (green) and $8 \mu \mathrm{m}$ (red). The blue and red contours represent the integrated intensity of the $\mathrm{CO}(3-2)$ transition in the blue- and red-shifted wings respectively. Contour levels are as in Fig. 2a. The yellow cross shows the position of the $\mathrm{CH}_{3} \mathrm{OH}$ maser spot, the circle the compact HII region (Walsh et al. 1998).

where no IRAC emission is detected, we actually believe the latter to be the case. However, we included the fluxes in the IRAC bands in Fig. 4, integrated over the APEX beam. The cm fluxes are from the data presented by Zapata et al. (2008); they correspond to the sum of fluxes of their sources VLA2a-d, restored with a beam of $\leq 1^{\prime \prime}$. An important difference between the VLA, APEX and IRAS observations is the spatial resolution, which is high in the $\mathrm{cm}$ region and coarse at the shorter wavelengths. From the source sizes derived from the analysis of the submm continuum (see Table 3), the emission detected with APEX is not confined to a compact source of a few arcsec or less, as the VLA sources, but it is rather extended, and probably contaminated by emission from components other than the hot core, e.g., a surrounding envelope. Therefore, one should regard the flux measures from APEX as upper limits to the fluxes of the compact hot core associated with the $\mathrm{cm}$ free-free emission.

\subsection{Outflow}

Figure 2a shows the map of the molecular outflow originating from IRAS 17233 traced by the $\mathrm{CO}(3-2)$ line; plotted are the intensities integrated over the velocity ranges covered by velocities lower and higher than the systematic velocity, i.e., red- and blue-shifted emission, respectively. Although the spectrum at the central position (see Fig. 1a) has both red and blue-shifted emission at high velocities, implying that the inclination angle of the flow with respect to the line of sight is small, the integrated intensity of the $\mathrm{CO}$ and $\mathrm{HCO}^{+}$lines in the wings shows a bipolar distribution: these two properties suggest a medium value for the inclination angle of the outflow. The flow has a north-south orientation, with the blue-shifted emission towards the north, and the red-shifted emission to the south. Strong emission at $4.5 \mu \mathrm{m}$, which is often interpreted as due to outflows (e.g., NoriegaCrespo et al. 2004), is detected along the direction of the molecular outflow (Fig. 5).

From the CO map it is clear that the lobes are barely resolved by our observations, and that higher resolution is needed to
Table 4. Parameters of the $\mathrm{CO}$ molecular outflow.

\begin{tabular}{lr}
\hline \hline$M_{\mathrm{tot}}\left[M_{\odot}\right]$ & 0.8 \\
$p\left[M_{\odot} \mathrm{km} \mathrm{s}^{-1}\right]$ & 25.3 \\
$E\left[10^{45} \mathrm{erg}\right]$ & 8.6 \\
$t[\mathrm{yr}]$ & 6200 \\
$\dot{M}_{\mathrm{tot}}\left[M_{\odot} \mathrm{yr}^{-1}\right]$ & 0.005 \\
$F_{\mathrm{m}}\left[M_{\odot} \mathrm{km} \mathrm{s}^{-1} \mathrm{yr}^{-1}\right]$ & 0.04 \\
$L_{\mathrm{m}}\left[L_{\odot}\right]$ & 48 \\
\hline
\end{tabular}

determine parameters such as the collimation factor and the opening and inclination angles of the outflow. The position angle of the flow, computed from the peak positions of the $\mathrm{CO}$ integrated emission in the two lobes, is close to $0^{\circ}$.

To compute the physical parameters of the outflowing gas we followed the standard formalism described by Cabrit \& Bertout (1990). The momentum $(p)$, the energy $(E)$, and the characteristic time scale $(t)$ can be derived assuming that the outflow is a constant velocity flow with true velocity equal to the maximum velocity observed. For the estimate of the mass in the lobes, we used a constant value for the integrated intensity of the CO line equal to its mean value computed on each lobe starting from the $5 \sigma$ level, and assumed that this is indeed representative of the flow region. To avoid contamination from the core emission, the $\mathrm{H}_{2}$ column density was obtained assuming a relative abundance of $\mathrm{CO}$ to molecular hydrogen of $10^{-4}$ and integrating between 9 and $50 \mathrm{~km} \mathrm{~s}^{-1}$ for the red-shifted gas, and -50 to $-10 \mathrm{~km} \mathrm{~s}^{-1}$ for the blue-shifted gas, which results in lower limits to the derived masses. Moreover, part of the red lobe emission is not covered by our observations. Since similar studies on molecular outflows from massive young stellar objects found gas in the flow at relatively high temperatures (Beuther et al. 2002a; Leurini et al. 2006), we assume that the excitation temperature of the outflow gas is $50 \mathrm{~K}$. Some authors (e.g., Bontemps et al. 1996) apply a multiplication factor of 3.5 for optical depth effects and a mean inclination angle of $57^{\circ} .3$ in computing the outflow parameters. Since the orientation of the outflow cannot be determined from our data, we decided not to correct for the inclination angle of the outflow. Moreover, observations of $\mathrm{CO}(3-2)$ and ${ }^{13} \mathrm{CO}(3-2)$ towards other massive flows show that the ratio of these two lines may change considerably from object to object (e.g., Leurini et al. 2006; Garay et al. 2007) thus implying differences in the optical depth of CO. The values reported in Table 4 should be considered as lower limits to the true values as 1) no corrections for the optical depth or inclination angle were applied; 2) the gas at low velocities was not considered in the calculations.

Beuther et al. (2002c) compared the physical properties of high-mass outflows to those of low-mass flows by studying the correlations between the mass and the luminosity of the core as function of the properties of the flow. The flow in IRAS 17233 has physical parameters characteristic of molecular flows driven by high-mass young stellar objects. In particular, the values derived for the mass entrainment rate $\dot{M}_{\text {tot }}$ and for the mechanical force $F$ are in agreement with a relatively low luminosity $\left(L \sim 10^{4} L_{\odot}\right)$ for the powering force of the flow.

\subsection{Molecular core}

The analysis of the molecular spectrum of IRAS 17233 was carried out with the XCLASS program (discussed by Comito et al. 2005), which uses an LTE model to produce synthetic spectra, and compares them to the observations. The molecular data are 
from the CDMS (Müller et al. 2001) and JPL (Pickett et al. 1998) databases. The parameters defining the synthetic spectrum are: source size, rotation temperature, column density, velocity offset and the velocity width. In our case, we did not use any offset in velocity, but adopted the systemic velocity of the source $\left(v_{\text {LSR }}-3.46 \mathrm{~km} \mathrm{~s}^{-1}\right)$ for all molecular species. Several velocity components, which are supposed to be non-interacting (i.e. the intensities add up linearly), can be used. The program can also produce double side band synthetic spectra for comparison against the observations. This is needed to model our data, since their frequency coverage does not permit sideband deconvolution. The galactocentric distance of the source was computed by Chin et al. (1996), and be found equal to $7.8 \mathrm{kpc}$. Following Wilson \& Rood (1994), the isotopic ratio ${ }^{12} \mathrm{C} /{ }^{13} \mathrm{C}$ at this distance is equal to 66, very close to the standard value of 60 used in XCLASS, and well within the errors of this estimate.

We started our analysis with the modelling of the $\mathrm{CH}_{3} \mathrm{CN}$ spectrum because several transitions are detected at once, providing good constraints on the physics of the gas they trace. The corresponding $\mathrm{CH}_{3}^{13} \mathrm{CN}$ band also falls within the bandwidth of our observations; however, at the rest frequencies of these lines, blending with other molecular species is severe and it is difficult to assess whether some of the lines are detected or not. However, even their non-detection would give us useful upper limits on the column density of this species. The best fit was found for a source size of $2.7^{\prime \prime}$, a temperature of $150 \mathrm{~K}$ and a column density of $\sim 4 \times 10^{16} \mathrm{~cm}^{-2}$.

Wilner et al. (1995) noticed that for luminosities typical of massive young stars, the temperature distribution in spherical, centrally illuminated dust clouds can be well approximated by the relation

$T_{\mathrm{d}}(R)=37\left(\frac{L}{L_{\odot}}\right)^{0.25}\left(\frac{R}{100 \mathrm{AU}}\right)^{-0.4}[\mathrm{~K}]$,

which relates the temperature of the dust $\left(T_{\mathrm{d}}\right)$ at a given distance $(R)$ to the luminosity $(L)$ of the inner source. Using this equation, and assuming local thermal equilibrium between dust and gas, we can independently derive the typical angular size for the hot core in IRAS 17233. Hughes \& MacLeod (1993) estimated an IR luminosity for the source of $1.5 \times 10^{5} L_{\odot}$, using a distance of $2.2 \mathrm{kpc}$. This estimate decreases to $\sim 2.5 \times 10^{4} L_{\odot}$ when adopting a distance of $1 \mathrm{kpc}$ (MacLeod et al. 1998), a value similar to that of Faúndez et al. (2004), who derived a bolometric luminosity of $1.4 \times 10^{4} L_{\odot}$ with a distance of $0.8 \mathrm{kpc}$.

Assuming a luminosity of $\sim 1.4 \times 10^{4} L_{\odot}$, the diameter of the region at $150 \mathrm{~K}$ is $2700 \mathrm{AU}$ for IRAS 17233 (see discussion in Sect. 4.4), which corresponds to $2.5^{\prime \prime}$ at a distance of $1 \mathrm{kpc}$, in agreement with the value derived through the analysis of the molecular spectrum.

For the best fit values, most of the $\mathrm{CH}_{3} \mathrm{CN}$ lines are optically thick; this implies a degeneracy in the model between the source size and the temperature. To derive the $3 \sigma$ confidence levels in the parameters, we fixed the source size to the best value derived in our analysis, and performed a $\chi^{2}$ analysis for the other two parameters in the spectral window between 294.0 and $294.3 \mathrm{GHz}$, which contains the $\mathrm{CH}_{3} \mathrm{CN}$ and $\mathrm{CH}_{3}^{13} \mathrm{CN}\left(16_{K}-15_{K}\right)$ lines. We found that the $3 \sigma$ confidence level for the kinetic temperature of the gas and the column density ranges between 114 and $290 \mathrm{~K}$, and $2 \times 10^{16}$ and $6 \times 10^{16} \mathrm{~cm}^{-2}$, respectively. We performed the same analysis on the torsionally excited lines of methanol. Although excitation by collisions is not an efficient way to populate these levels, which are pumped through the infrared radiation field (Leurini et al. 2007), their populations are easily
Table 5. Molecular parameters for IRAS 17233.

\begin{tabular}{|c|c|c|c|c|}
\hline Species & $\begin{array}{c}\text { Size } \\
\left({ }^{\prime \prime}\right)\end{array}$ & $\begin{array}{l}T_{\mathrm{ex}} \\
(\mathrm{K})\end{array}$ & $\begin{array}{c} \\
\left(\mathrm{cm}^{-2}\right)\end{array}$ & Comment \\
\hline $\mathrm{C}_{2} \mathrm{H}_{5} \mathrm{CN}$ & $2.7^{a}$ & $150^{a}$ & $5(16)$ & $b$ \\
\hline $\mathrm{CH}_{3} \mathrm{CCH}$ & ext. $^{a}$ & 100 & $2(15)$ & ${ }^{c}$ \\
\hline $\mathrm{CH}_{3} \mathrm{CN}$ & 2.7 & 150 & $4(16)$ & \\
\hline $\mathrm{CH}_{3} \mathrm{OCHO}-\mathrm{a}$ & $2.7^{a}$ & $150^{a}$ & $2(17)$ & $b$ \\
\hline $\mathrm{CH}_{3} \mathrm{OCHO}-\mathrm{e}$ & $2.7^{a}$ & $150^{a}$ & $2(17)$ & $b$ \\
\hline \multirow[t]{2}{*}{$\mathrm{CH}_{3} \mathrm{OH}$} & $2.7^{a}$ & $150^{a}$ & $2(18)$ & $d$ \\
\hline & ext. $^{a}$ & 40 & $8(15)$ & \\
\hline $\mathrm{CO}$ & ext. $^{a}$ & 30 & $5(19)$ & e \\
\hline CS & ext. $^{a}$ & 30 & $5(15)$ & $c$ \\
\hline DCN & ext. $^{a}$ & 150 & $4(13)$ & $c$ \\
\hline $\mathrm{H}_{2} \mathrm{CO}$ & ext. $^{a}$ & 80 & $2(15)$ & c \\
\hline $\mathrm{H}_{2} \mathrm{CS}$ & ext. $^{a}$ & 70 & $9(14)$ & c \\
\hline \multirow[t]{2}{*}{$\mathrm{HC}_{3} \mathrm{~N}$} & $2.7^{a}$ & $150^{a}$ & $3(16)$ & \\
\hline & ext. $^{a}$ & 50 & $9(15)$ & \\
\hline $\mathrm{HNCO}$ & $2.7^{a}$ & $150^{a}$ & $3(16)$ & c \\
\hline $\mathrm{SO}$ & ext. $^{a}$ & 40 & $3(15)$ & \\
\hline $\mathrm{SO}_{2}$ & ext. $^{a}$ & 60 & $5(15)$ & \\
\hline
\end{tabular}

${ }^{a}$ Fixed parameter; ${ }^{b}$ based on weak or partially blended lines only; ${ }^{c}$ based on one or a few lines only; ${ }^{d}$ includes vibrationally excited lines; ${ }^{e}$ based on $\mathrm{C}^{17} \mathrm{O}(3-2)$ only.

thermalised at the temperature of the dust by the dust radiation field. Since it can be assumed that dust and gas are in thermal equilibrium (Krügel \& Walmsley 1984) at the high density of the inner region around massive protostars, we believe that the LTE approach, defined by one temperature for the dust and for the gas, is reasonable also for the $v_{t}=1$ lines of methanol. The minimum of the $\chi^{2}$ distribution is found for different values than the ones delivered by the $\mathrm{CH}_{3} \mathrm{CN}$ analysis $\left(T \sim 210 \mathrm{~K}, s \sim 1.7^{\prime \prime}\right.$, $N \sim 9 \times 10^{18} \mathrm{~cm}^{-2}$ ). However, this model offers a good fit for the higher energy levels in the band, while the other lines have high optical depths and their profiles do not match the observations. Therefore, in Table 5 we list the column density obtained for a source size and a temperature equal to that obtained from the $\mathrm{CH}_{3} \mathrm{CN}$ analysis, which still fall in the $3 \sigma$ confidence level of the $\chi^{2}$ distribution of $\mathrm{CH}_{3} \mathrm{OH}$. In Fig. 6, we present an example of the quality of the fit for $\mathrm{CH}_{3} \mathrm{OH} v_{t}=1$ and $\mathrm{CH}_{3} \mathrm{CN}$ lines. The other spectral windows, and the synthetic spectrum corresponding to the best fit model, are shown in Fig. 8 in the Online Material section.

The parameters used in the model for the different molecular species are shown in Table 5. The emission of several molecular species is compatible with that of a compact source with the temperature and source size derived from acetonitrile. These are complex molecules, whose abundances are enhanced by thermal evaporation of grain mantles, or whose high excitation lines are efficiently excited only by infrared pumping. In the remaining cases, the source was assumed to be extended, and the column density given in Table 5 corresponds to the beam averaged column density. This approach is justified for optically thin emission, where the source size and the column density are degenerate parameters of the model. However, it should be noticed that this approach was used mostly for molecules where only a limited number of transitions were available.

\subsection{Comparison with other hot cores}

Comparison between hot cores associated with sources of different luminosities can help to understand whether their chemical composition is a function of the mass and luminosity of the 

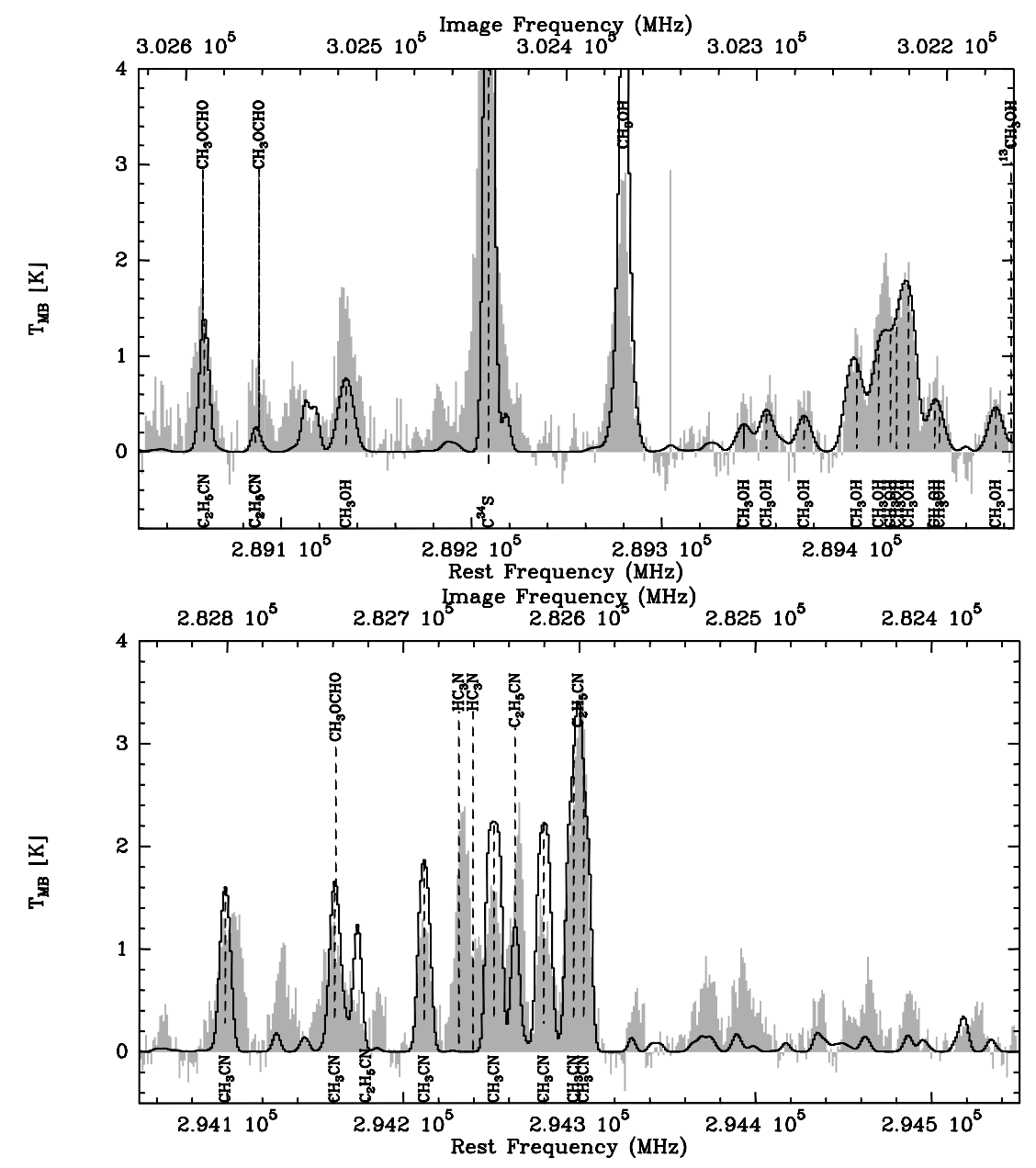

Fig. 6. Molecular emission from IRAS 17233-3606. The solid line represents the synthetic spectrum obtained for the best fit solution.

central heating source, whether it simply reflects the evolutionary state of the source and its initial conditions.

The assumed luminosity of IRAS 17233 is $\sim 1.4 \times 10^{4} L_{\odot}$. For NGC 6344(I) and for G327.3-0.6, attempts to derive their bolometric luminosity were made by Sandell (2000) and Wyrowski et al. (2006) respectively, who report values of $2.6 \times 10^{5} L_{\odot}$ for NGC 6334(I), and 5-15 × $0^{4} L_{\odot}$ for G327.3-0.6. Therefore, IRAS 17233 is the least powerful of the three hot cores we observed. On the other hand, it is likely to be the closest among these sources $\left(D_{\mathrm{G} 327.03} \sim 2.9 \mathrm{kpc}, D_{\mathrm{NGC6334(I)}} \sim 1.7 \mathrm{kpc}\right.$, Bergman 1992; Neckel 1978), and it could have the largest beam filling factor in our observations. This would explain its exceptionally strong molecular spectrum despite its relatively low luminosity.

However, using Eq. (1), we found that the size of the region at $150 \mathrm{~K}$ is equal to $2700 \mathrm{AU}$ for IRAS $17233\left(\sim 2.5^{\prime \prime}\right)$, $15000 \mathrm{AU}$ for NGC 6334(I) ( 8 $\left.8^{\prime \prime}\right)$, and, given its luminosity uncertainty, between 5200 and 10000 AU ( 1.7" and 3.4", respectively) for G327.3-0.6. According to these results, the exceptionally strong molecular spectrum of IRAS 17233 cannot be attributed to a beam filling factor larger than for the other sources. This would rather suggest that the strength of the lines from IRAS 17233 are due to an intrinsic property of the source. However, the source sizes derived through Eq. (1) are affected by the uncertainties in the distance, which at least in the case of IRAS 17233 is high, and in the luminosity, which for all sources is based on relatively low resolution data.
Schilke et al. (2006) and Comito et al. (2005) studied the hot cores in NGC 6334(I), G327.3-0.6 and Orion-KL with the same technique used for IRAS 17233, although at different frequencies. Therefore, the results of these analyses are affected by the same systematic errors of the method, and comparison with our results should be straightforward. However, the source size used in their studies is not estimated from observations but derived from the models. Since this parameter is degenerate with the temperature for optically thick lines, and with the temperature and the column density for optically thin lines, the column densities derived for the hot cores are strongly dependent on the source size used in the modelling. To cancel out this effect, we compared relative abundances in the different sources. Given the different spectral windows analysed, only $\mathrm{CH}_{3} \mathrm{CN}$ is observed in all hot cores. Therefore, we used $\mathrm{CH}_{3} \mathrm{CN}$ as reference molecule to compute the abundances of the other species. The species associated with the hot cores, and in common between different sources, are $\mathrm{CH}_{3} \mathrm{OH}$ and $\mathrm{HNCO}$ (in IRAS 17233, NGC 6334(I), Orion-KL), $\mathrm{C}_{2} \mathrm{H}_{5} \mathrm{CN}$ (in IRAS 17233, Orion-KL, G327.3-0.6), and $\mathrm{CH}_{3} \mathrm{OCHO}$ (in IRAS 17233, NGC 6334(I), G327.3-0.6). Moreover, Schöier et al. (2002) studied the molecular content of the hot core around the solar type protostar IRAS 16293-2422, and derived abundances for four of the species analysed here $\left(\mathrm{CH}_{3} \mathrm{CN}, \mathrm{CH}_{3} \mathrm{OH}, \mathrm{HNCO}\right.$ and $\left.\mathrm{CH}_{3} \mathrm{OCHO}\right)$.

By comparing the results for these five sources, we could not find any trend in the abundances as function of the luminosity of the object, and all sources show different values for each species. However, for all sources the most abundant species 


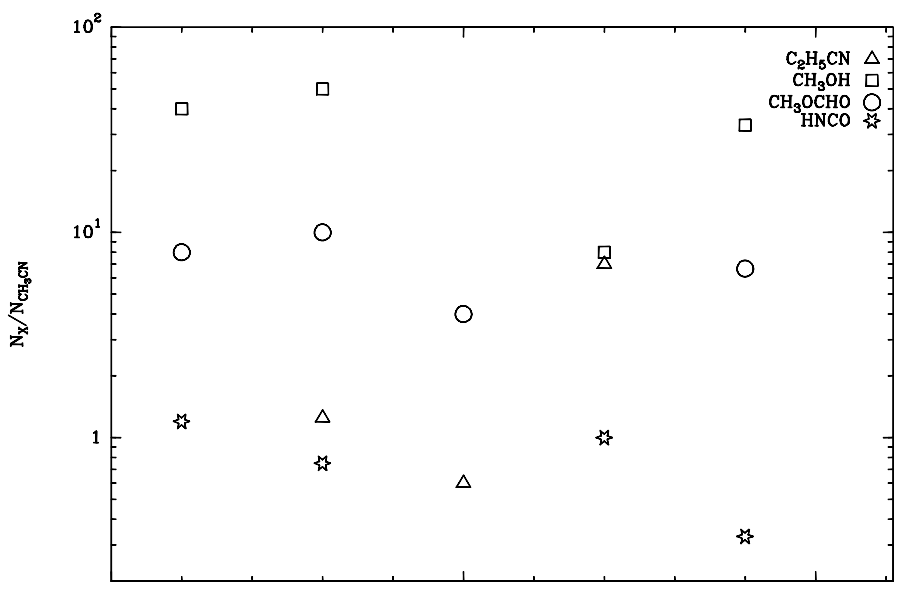

16293-2422 $17233-3606 \quad$ G327.3-0.6 $\quad$ ORION-KL NGC 6334(I)

Fig. 7. Comparison of molecular abundances of $\mathrm{CH}_{3} \mathrm{OH}, \mathrm{HNCO}$, $\mathrm{CH}_{3} \mathrm{OCHO}$ and $\mathrm{C}_{2} \mathrm{H}_{5} \mathrm{CN}$ respect to $\mathrm{CH}_{3} \mathrm{CN}$.

is $\mathrm{CH}_{3} \mathrm{OH}$, followed in by $\mathrm{CH}_{3} \mathrm{OCHO}, \mathrm{C}_{2} \mathrm{H}_{5} \mathrm{CN}$ and $\mathrm{HNCO}$. For IRAS 16293-2422, the abundance of $\mathrm{CH}_{3} \mathrm{OCHO}$ is an upper limit. Figure 7 presents an overview of the molecular abundances with respect to $\mathrm{CH}_{3} \mathrm{CN}$ in the five sources. Hatchell et al. (1998a) performed a similar analysis on a larger sample of hot cores, and found no systematic difference between their sources. However, we did not include their results in our analysis because for most of the molecular species they are limits to the column densities.

We believe that such a comparison of the chemistry of hot cores associated with heating objects of different mass and luminosity, and including hot corinos around low mass protostars, would indeed be useful for a better understanding of this evolutionary stage. However, we stress that this analysis should be performed with the same method and on observations of the same frequency windows and linear resolutions to cancel out all systematic errors.

Figure 3 presents the molecular spectrum at $\sim 294 \mathrm{GHz}$ of the three hot cores IRAS 17233, NGC 6344(I) and G327.30.6. The spectra are relatively similar, although NGC 6334(I) and G327.3-0.6 show stronger emission than IRAS 17233 in the ${ }^{13} \mathrm{CH}_{3} \mathrm{OH}\left(6_{K}-5_{K}\right) v_{t}=0,1$ band, while the $\mathrm{CH}_{3} \mathrm{CN}\left(16_{K}-15_{K}\right)$ is more intense in IRAS 17233, at least in its lower transitions lines. However, a striking difference between the three sources is in the intensity of the $\mathrm{SO}_{2}$ lines (and $\mathrm{SO}$ transitions at other frequencies), which are by far stronger in IRAS 17233 than in the other two cores. The abundance of S-bearing molecules can be enhanced by thermal evaporation of icy grain mantles due to protostellar heating (e.g., Charnley 1997), or by shocks (e.g., Mitchell 1984). Among other hot cores, Orion-KL is known to be exceptionally strong in $\mathrm{SO}$ and $\mathrm{SO}_{2}$ (e.g., Schilke et al. 1997), which are associated with a molecular outflow (Wright et al. 1996). In the case of IRAS 17233, the linewidths of the $\mathrm{SO}$ and $\mathrm{SO}_{2}$ transitions are broad (9-12 $\mathrm{km} \mathrm{s}^{-1}$ ), but reasonably fitted with single Gaussian profiles, suggesting that the bulk of the emission does not arise in the outflow. However, for two of the $\mathrm{SO}_{2}$ transitions a two component Gaussian fit gives a better agreement with the observations. Both transitions have asymmetric profiles, with non-Gaussian blue-shifted emission. Figure $1 \mathrm{~b}$ shows one of the two asymmetric $\mathrm{SO}_{2}$ transitions, which is in the same frequency setup of the $\mathrm{HCO}^{+}(4-3)$ observations, but comes from the other side band. The integrated emission of this line is compact, but slightly elongated along the axis of the molecular outflow. However, only a map of the region in these two molecular species at higher angular resolution would unambiguously associate their emission with the hot core or with the outflow.

Hatchell et al. (1998b) and van der Tak et al. (2003) studied several massive star-forming regions in different $\mathrm{S}$-bearing molecules, and found that the abundances of $\mathrm{SO}$ and $\mathrm{SO}_{2}$ are the ones with the largest spread from source to source. van der Tak et al. (2003) also found that up to $50 \%$ of the $\mathrm{SO}$ and $\mathrm{SO}_{2}$ emission arises in high-velocity gas. No trend in their column densities with temperature was found. These studies agree on the fact that the abundances of $\mathrm{SO}$ and $\mathrm{SO}_{2}$ are strongly dependent on the source, which suggests that they are related either to its evolutionary stage or to its initial conditions as already suggested by chemical models (e.g., Charnley 1997 for the dependence on the evolutionary stage, Wakelam et al. 2004 for the dependence on the initial conditions).

\section{Conclusion}

We observed the massive star-forming region IRAS 17233-3606 with the APEX telescope in several molecular transitions of high diagnostic value at a wavelength of $1 \mathrm{~mm}$. Our observations reveal a bipolar molecular outflow associated with strong emission at $4.5 \mu \mathrm{m}$, and an exceptionally rich molecular spectrum from a position very close to the centre of the outflow. To better characterise the nature of this source, we performed continuum crossscans on the source at $0.9,0.7$ and $0.4 \mathrm{~mm}$, and derived the flux density of the source over a beam between 18 and 7.6".

We modelled the molecular emission of the source using a LTE approximation, and found that it is compatible with that of a hot core of $150 \mathrm{~K}$ and a source size of $2.7^{\prime \prime}$. The distance of the source, and its bolometric luminosity, are not well known. However, assuming that the source is at a distance of $1 \mathrm{kpc}$, and that the bulk of the dust is at $70 \mathrm{~K}$, we found that its submillimetre continuum emission corresponds to a mass in the range of 103-240 $M_{\odot}$. We also analysed the molecular emission associated with the bipolar outflow, using the $\mathrm{CO}$ (3-2) transition and assuming that the emission is optically thin and the gas is at $50 \mathrm{~K}$. The physical parameters are typical of those driven by high-mass young stellar objects. However, follow-up observations in high energy and in low optically depth transitions are needed for a better characterisation of the outflow.

If the estimate of the distance to the source is correct, IRAS $17233-3606$ is probably placed among the less studied hot cores around B-type (proto)stars. This seems to be confirmed by the parameters of the molecular outflow, which put its powering source among the less luminous massive sources. It is therefore an interesting target for future high-angular resolution observations aimed at investigating the connection between hot cores around objects of high-mass and hot corinos found around solar type protostars. Moreover, given its close distance and its location in sky, IRAS 17233 will easily be accessible in the near future for high linear resolution studies with ALMA.

Acknowledgements. We would like to thank Leonardo Testi and Bernd Klein for helpful discussions. We appreciate the careful referee's report which helped improving the paper.

\section{References}

Benjamin, R. A., Churchwell, E., Babler, B. L., et al. 2003, PASP, 115, 953 Bergman, P. 1992, Ph.D. Thesis, Göteborg, Sweden

Beuther, H., Schilke, P., Gueth, F., et al. 2002a, A\&A, 387, 931

Beuther, H., Schilke, P., Menten, K. M., et al. 2002b, ApJ, 566, 945 
Beuther, H., Schilke, P., Sridharan, T. K., et al. 2002c, A\&A, 383, 892 Bontemps, S., Andre, P., Terebey, S., \& Cabrit, S. 1996, A\&A, 311, 858 Cabrit, S. \& Bertout, C. 1990, ApJ, 348, 530

Carey, S. J., Noriega-Crespo, A., Price, S. D., et al. 2005, BAAS, 37, 1252 Charnley, S. B. 1997, ApJ, 481, 396

Chin, Y.-N., Henkel, C., Whiteoak, J. B., Langer, N., \& Churchwell, E. B. 1996 A\&A, 305, 960

Comito, C., Schilke, P., Phillips, T. G., et al. 2005, ApJS, 156, 127

De Buizer, J. M., Piña, R. K., \& Telesco, C. M. 2000, ApJS, 130, 437

Faúndez, S., Bronfman, L., Garay, G., et al. 2004, A\&A, 426, 97

Fish, V. L., Reid, M. J., Argon, A. L., \& Zheng, X.-W. 2005, ApJS, 160, 220

Fix, J. D., Mutel, R. L., Gaume, R. A., \& Claussen, M. J. 1982, ApJ, 259, 657

Forster, J. R., \& Caswell, J. L. 1989, A\&A, 213, 339

Garay, G., Mardones, D., Bronfman, L., et al. 2007, A\&A, 463, 217

Güsten, R., Nyman, L. A.., Schilke, P., et al. 2006, A\&A, 454, L13

Hatchell, J., Thompson, M. A., Millar, T. J., \& MacDonald, G. H. 1998a, A\&AS, 133,29

Hatchell, J., Thompson, M. A., Millar, T. J., \& MacDonald, G. H. 1998b, A\&A, 338,713

Hatchell, J., Fuller, G. A., Millar, T. J., Thompson, M. A., \& Macdonald, G. H. 2000, A\&A, 357, 637

Heyminck, S., Kasemann, C., Güsten, R., de Lange, G., \& Graf, U. U. 2006, A\&A, 454, L21

Hieret, C., Leurini, S., Menten, K. M., et al. 2007, ArXiv e-prints, 706

Hildebrand, R. H. 1983, QJRAS, 24, 267

Hughes, V. A., \& MacLeod, G. C. 1993, AJ, 105, 1495

Klein, B., Philipp, S. D., Krämer, I., et al. 2006, A\&A, 454, L29

Krügel, E., \& Walmsley, C. M. 1984, A\&A, 130, 5

Leurini, S., Schilke, P., Menten, K. M., et al. 2004, A\&A, 422, 573

Leurini, S., Schilke, P., Parise, B., et al. 2006, A\&A, 454, L83

Leurini, S., Schilke, P., Wyrowski, F., \& Menten, K. M. 2007, A\&A, 466, 215

Müller, H. S. P., Thorwirth, S., Roth, D. A., \& Winnewisser, G. 2001, A\&A, 370, L49

MacLeod, G. C., Scalise, E. J., Saedt, S., Galt, J. A., \& Gaylard, M. J. 1998, AJ, 116,1897

Miettinen, O., Harju, J., Haikala, L. K., \& Pomrén, C. 2006, A\&A, 460, 721

Mitchell, G. F. 1984, ApJ, 287, 665

Molinari, S., Brand, J., Cesaroni, R., \& Palla, F. 2000, A\&A, 355, 617
Molinari, S., Brand, J., Cesaroni, R., Palla, F., \& Palumbo, G. G. C. 1998, A\&A, 336,339

Molinari, S., Brand, J., Cesaroni, R., \& Palla, F. 1996, A\&A, 308, 573

Neckel, T. 1978, A\&A, 69, 51

Noriega-Crespo, A., Morris, P., Marleau, F. R., et al. 2004, ApJS, 154, 352

Olmi, L., Cesaroni, R., \& Walmsley, C. M. 1993, A\&A, 276, 489

Ossenkopf, V. \& Henning, T. 1994, A\&A, 291, 943

Osterloh, M., Henning, T., \& Launhardt, R. 1997, ApJS, 110, 71

Pickett, H. M., Poynter, I. R. L., Cohen, E. A., et al. 1998, J. Quant. Spectr. Rad. Trans., 60, 883

Risacher, C., Vassilev, V., Monje, R., et al. 2006, A\&A, 454, L17

Sandell, G. 2000, A\&A, 358, 242

Schilke, P., Comito, C., Thorwirth, S., et al. 2006, A\&A, 454, L41

Schilke, P., Groesbeck, T. D., Blake, G. A., \& Phillips, T. G. 1997, ApJS, 108, 301

Schöier, F. L., Jørgensen, J. K., van Dishoeck, E. F., \& Blake, G. A. 2002, A\&A, 390, 1001

Sridharan, T. K., Beuther, H., Schilke, P., Menten, K. M., \& Wyrowski, F. 2002, ApJ, 566, 931

van der Tak, F. F. S., Boonman, A. M. S., Braakman, R., \& van Dishoeck, E. F. 2003, A\&A, 412, 133

Wakelam, V., Caselli, P., Ceccarelli, C., Herbst, E., \& Castets, A. 2004, A\&A, 422,159

Walsh, A. J., Hyland, A. R., Robinson, G., \& Burton, M. G. 1997, MNRAS, 291, 261

Walsh, A. J., Burton, M. G., Hyland, A. R., \& Robinson, G. 1998, MNRAS, 301, 640

Walsh, A. J., Burton, M. G., Hyland, A. R., \& Robinson, G. 1999, MNRAS, 309, 905

Williams, S. J., Fuller, G. A., \& Sridharan, T. K. 2004, A\&A, 417, 115

Williams, S. J., Fuller, G. A., \& Sridharan, T. K. 2005, A\&A, 434, 257

Wilner, D. J., Welch, W. J., \& Forster, J. R. 1995, ApJ, 449, L73

Wilson, T. L., \& Rood, R. 1994, ARA\&A, 32, 191

Wright, M. C. H., Plambeck, R. L., \& Wilner, D. J. 1996, ApJ, 469, 216

Wyrowski, F., Menten, K. M., Schilke, P., et al. 2006, A\&A, 454, L91

Zapata, L., Leurini, S., Menten, K. M., Schilke, \& R. R. 2008, submitted

Zhang, Q., Hunter, T. R., Brand, J., et al. 2001, ApJ, 552, L167

Zhang, Q., Hunter, T. R., Brand, J., et al. 2005, ApJ, 625, 864 
S. Leurini et al.: Star formation in the IRAS 17233-3606 region, Online Material $p 1$
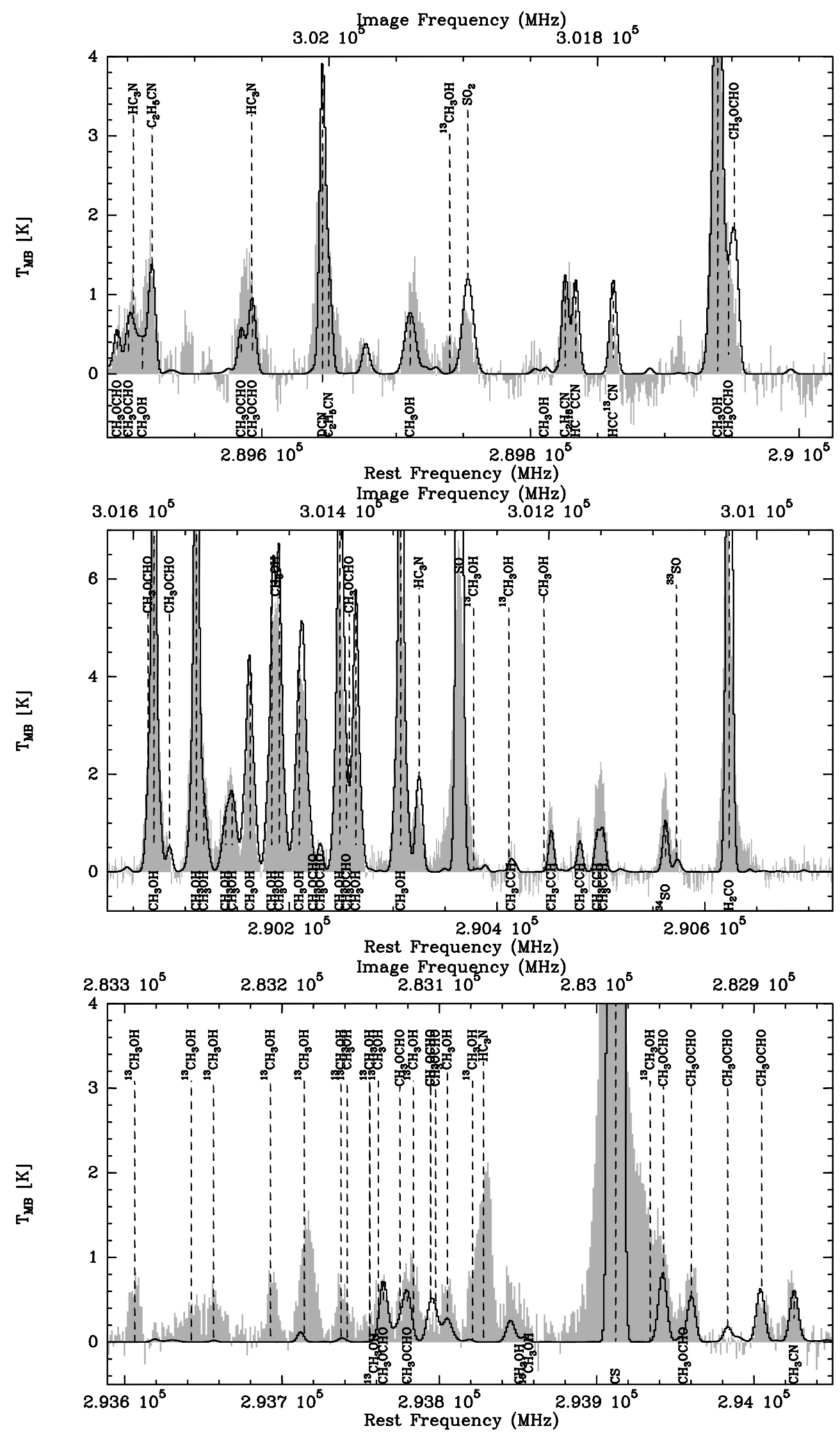

Fig. 8. Molecular emission from IRAS 17233-3606. The solid line represents the synthetic spectrum obtained for the best fit solution. 
S. Leurini et al.: Star formation in the IRAS 17233-3606 region, Online Material p 2
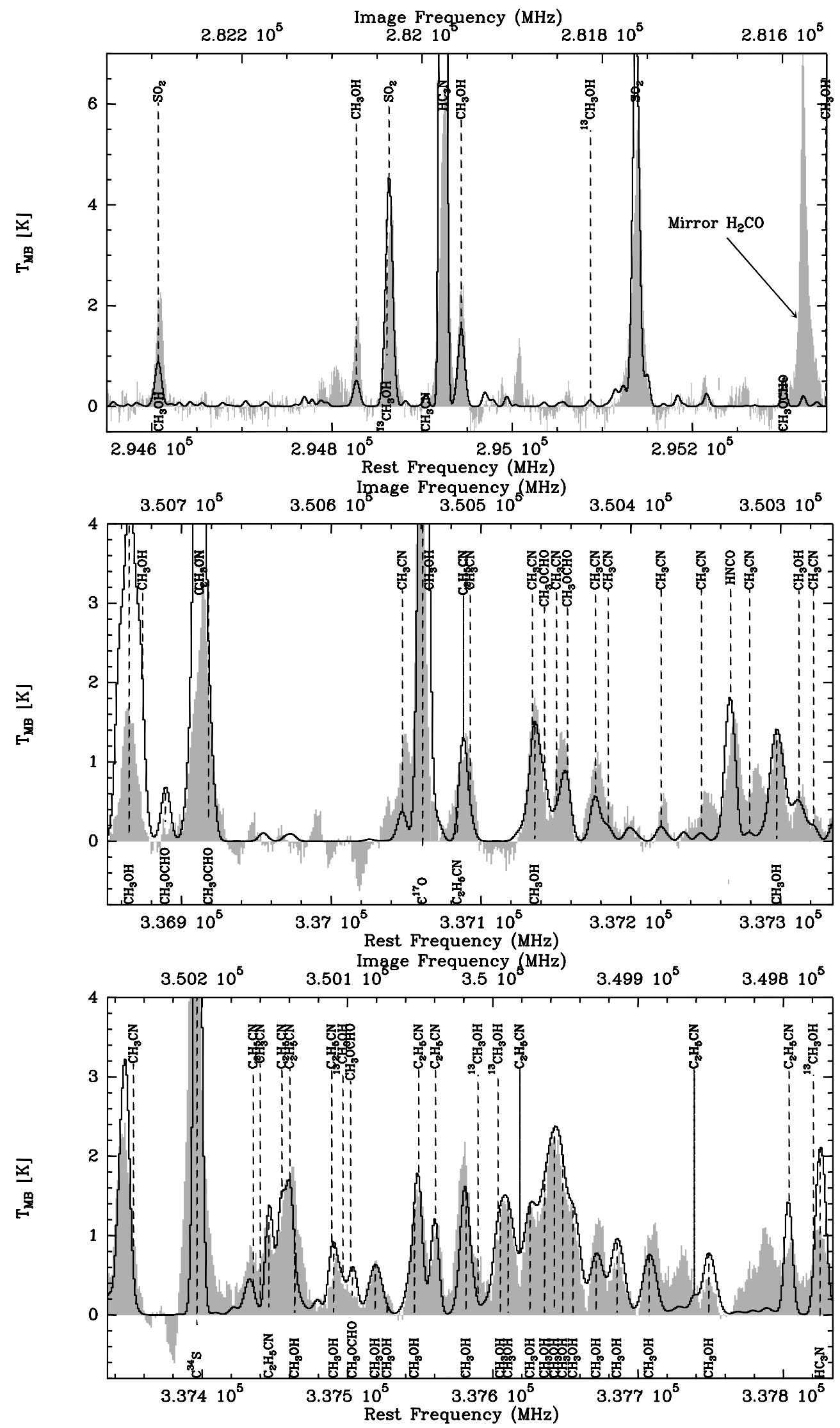

Fig. 8. continued. 
S. Leurini et al.: Star formation in the IRAS 17233-3606 region, Online Material p 3
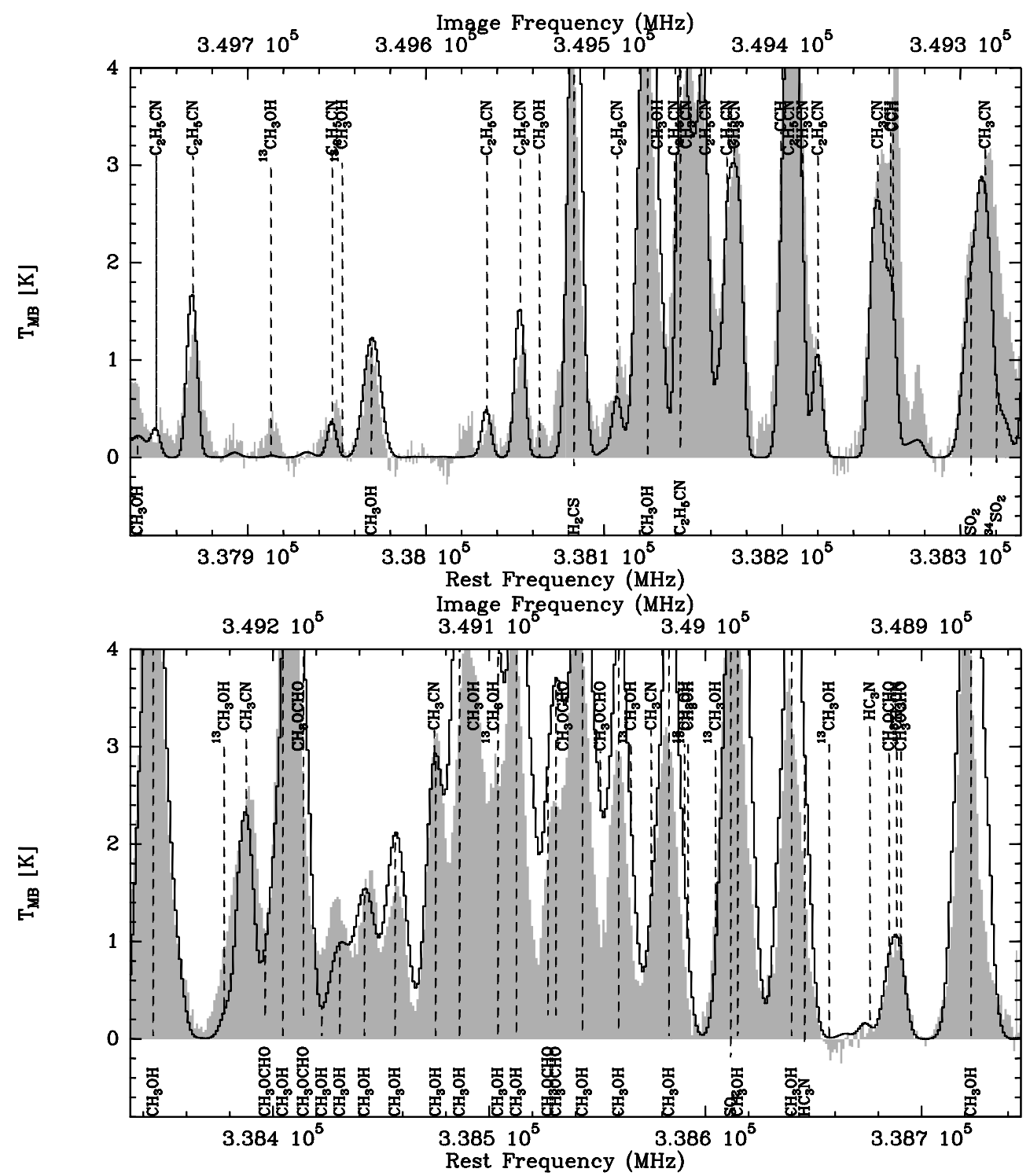

Fig. 9. continued. 